\title{
A COMPLEXIDADE DE CONSENSO ENTRE ANÁLISE SENSORIAL, FÍSICA E- QUÍMICA NA QUALIDADE DO CAFÉ
}

\section{RESUMO}

Lucas Louzada Pereira ${ }^{1}$ Wilton Soares Cardoso ${ }^{1}$ Rogério Carvalho Guarçoni ${ }^{2}$ Taís Rizzo Moreira ${ }^{3}$ Marina Gomes de Castro ${ }^{1}$ Patrícia Fontes Pinheiro ${ }^{4}$ Maria Amélia Gava Ferrão ${ }^{2}$ Aymbiré Francisco Almeida da Fonseca ${ }^{2}$

Carla Schwengster ten Caten ${ }^{5}$

O consenso para a definição qualitativa de um alimento não é uma tarefa trivial, entretanto, quando analisada a composição química e sensorial do café, a tarefa se torna ainda mais complexa. Recentes discussões no meio acadêmico retratam os esforços entre as tentativas de convergências entre o emprego de técnicas físicas e químicas em relação ao processo de análise sensorial de cafés especiais, visando entender e estimar parâmetros de processos. Dado que o método de avaliação da qualidade (análise sensorial) é realizado por Q-Graders, e que este processo pode ser passível de falhas durante a análise. Este estudo verificou o consenso entre o agrupamento dos cafés por meio da análise sensorial e análise física e química, através da técnica de componentes principais - PCA, para avaliar o quão próximo ou distante os resultados se agrupam na análise visual dos componentes. Como metodologia, utilizou-se o protocolo da Associação Americana de Cafés Especiais - SCAA, bem como análises físicas e químicas de extrato aquoso, cafeína, acidez titulável e pH. Os resultados indicam que dados oriundos das análises físicas e químicas não possuem o mesmo consenso em relação ao agrupamento observado nos dados das análises sensoriais dos cafés. A maneira pela qual os Q-Graders interpretam a qualidade se difere da forma como os dados químicos se agrupam, assim, os resultados sugerem que fatores além da composição física e química do café impactam na percepção de como a qualidade final é formulada.

\footnotetext{
${ }^{1}$ Instituto Federal de Educação, Ciência e Tecnologia do Espírito Santo - IFES Campus Venda Nova do Imigrante, Venda Nova do Imigrante - ES. Autor para correspondência: lucaslozada@hotmail.com.

${ }^{2}$ Instituto Capixaba de Pesquisa, Assistência Técnica e Extensão Rural - INCAPER.

${ }^{3}$ Universidade Federal do Espírito Santo - UFES Campus Jerônimo Monteiro, Centro de Ciências Agrárias e Engenharias.

${ }^{4}$ Universidade Federal do Espírito Santo - UFES Campus Alegre, Departamento de Agronomia.

${ }^{5}$ Universidade Federal do Rio Grande do Sul - UFRGS, Departamento de Engenharia de Produção e Transportes.
} 
Palavras-chave: Coffea arabica L. Análises sensoriais, físicas e químicas. Componentes Principais.

\title{
THE CONSENSUS COMPLEXITY BETWEEN SENSORY AND PHYSICAL-CHEMICAL ANALYSIS IN THE QUALITY OF COFFEE
}

\begin{abstract}
The consensus for the qualitative definition of the food is not a trivial task, however, when the chemical and sensorial composition of coffee is analyzed, the task becomes even more complex. Recent discussions, in the academic world, represent the efforts between the attempts of convergences among the use of physical and chemical techniques in relation to the process of sensorial analysis of specialty coffees, aiming to understand and estimate process parameters. Since that the quality evaluation method (sensory analysis) is performed by Q-Graders, this process may be subject to failures during the analysis. This study verified the consensus between the grouping of the coffees through the sensorial, physical and chemical analysis, through the technique of main components - PCA, to evaluate how close or distant the results are grouped in the visual analysis of the components. As a methodology, the protocol of the American Association of Special Coffees SCAA was used, as well as physical and chemical analysis of aqueous extract, caffeine, titratable acidity and $\mathrm{pH}$. The results indicate that data from the physical and chemical analysis do not have the same consensus regarding the grouping observed in the data of the sensorial analysis of the coffees. That is, the way Q-Graders interpret quality differs from the way the chemical data groups, so the results suggest that factors beyond the physical and chemical composition of coffee impact the perception of how the final quality is formulated.
\end{abstract}

Keywords: Coffea arabica L. Sensory analysis, physical chemistry. Principal Components.

\section{INTRODUÇÃO}

O Brasil é o maior produtor e exportador de café do mundo, a qualidade dos cafés brasileiros é conhecida mundialmente em detrimento das características sensoriais, dada as diversas condições climáticas, produtivas, seguido dos métodos de colheita, processamento, fermentação e secagem que são empregados nas propriedades (PEREIRA, 2017).

No comércio de café, os procedimentos da análise sensorial são utilizados para a negociação da commodity, tendo como base a qualidade da bebida, que é descrita pelos Q-Graders, usando opinião pessoal e experiência de degustação acumulada ao longo dos anos (FERIA-MORALE, 2002). 
Apesar do processo de análise sensorial ser amplamente utilizado, para DiDonfrancesco et al. (2014), este não se constitui no melhor método de avaliação da qualidade do café, devido a uma gama de fatores que interferem no processo de degustação.

Além disso, muitas vezes essa técnica tem alto custo e requer longo tempo de treinamento para se alcançar a concordância dos provadores para as interpretações dos termos que serão usados (SCHOLS et al., 2013). Para Bhumiratana, Adhikari e Chambers (2011), os componentes aromáticos são particularmente importantes em café especiais, e estes compostos são os principais constituintes da experiência sensorial de provadores de café.

São muitos os fatores que compõem a formação do sabor do café, a de se citar os constituintes químicos voláteis e não-voláteis, que para Moreira e Turgo (1999), são fatores que interferem nos processos sensoriais, sendo o aroma o mais complexo. Enquanto a sensação do sabor é relativamente simples, sendo normalmente classificada em cinco percepções (doce, salgado, amargo, umami e azedo), as modalidades de aroma ainda estão sendo intensamente discutidas nos meios científicos. Neste contexto, pesquisas conduzidas por Sáenz-Navajas et al. (2010) e Scholz et al. (2013), buscaram entender a relação entre composição química e qualidade final do café, com a finalidade de correlacionar tais constituintes, essas estratégias visam à amplificação de outras ferramentas e técnicas que possam auxiliar na tomada de decisão, de modo que se possa chegar a conclusões mais precisas sobre os aspectos relativos ao processo de análise sensorial de cafés, que é tido como algo subjetivo.

Especialistas em processos de análise sensorial discutem a necessidade de mesclar as técnicas de avaliação da qualidade, que utilizam Q-Graders, com métodos que considerem análises físicas e químicas. São referências os trabalhos de Pinto et al. (2001), Seo et al. (2008); Alvarado e Linnemann (2010), DonFrancesco et al. (2014), que recomendam tais combinações, buscando estabelecer possíveis relações entre o conteúdo de determinadas características físicas e químicas com as sensoriais, visando elevar o nível de acurácia na quantificação e descrição da qualidade de um café.

A oportunidade de sumarizar grandes grupos de dados, reduzindo sua dimensionalidade e permitindo interpretações conjuntas das variáveis envolvidas no estudo, apresenta-se como alternativa viável para a análise de resultados da avaliação da qualidade de grãos de café (CLEMENTE et al., 2015). 
Este estudo analisou e verificou o consenso entre o agrupamento dos cafés por meio da análise sensorial, seguida das análises físicas e químicas, com o objetivo de avaliar o quão próximo ou distante os resultados se agrupam na análise visual dos componentes principais - PCA.

\section{MATERIAIS E MÉTODOS}

Para avaliação sensorial, foram selecionadas 20 amostras de cafés oriundas de diversos pontos geográficos na região Serrana do Espírito Santo. Os materiais compostos para realização do estudo foram processados pelo método via-úmida, seguindo as proposições recomendadas por Pereira et al., (2018). As amostras foram preparadas no laboratório de análise e pesquisa em café - LAPC, do Instituto Federal do Espírito Santo, campus Venda Nova do Imigrante.

A primeira etapa constituiu-se pela análise sensorial, que foi realizada por 6 avaliadores utilizando a metodologia de análise sensorial, da Specialty Coffee Association of America, SCAA (2013). A segunda etapa constituiu-se das análises físicas e químicas, de extrato aquoso, cafeína, acidez titulável e $\mathrm{pH}$.

As torras foram conduzidas utilizando o torrador Laboratto TGP2 e os perfis de torra foram monitorados pelo conjunto de discos Agtron-SCAA, o ponto de torra destas amostras, situou-se entre as cores determinadas pelos discos \#65 e \#55 (SCAA, 2013).

As torras foram executadas com 24 horas de antecedência e a moagem respeitou o tempo de 8 horas de descanso após a torra. Todas as amostras foram torradas entre 8 a 10 minutos e, após a torra e o resfriamento, as amostras permaneceram lacradas, conforme a metodologia de análise sensorial descritiva. Para avaliação dos cafés, foi utilizado o protocolo da SCAA (2013).

\section{Preparo das amostras para degustação}

As 20 amostras de cafés foram moídas com moedor elétrico Bunn G3, com granulometria média/grossa. Cada lote de foi degustado com 5 xícaras, sendo adotada a concentração ótima 8,25 gramas de café moído em 150ml de água, em conformidade com o ponto médio do gráfico de equilíbrio ótimo para obtenção do Golden Cup (SCAA, 2013). O ponto de infusão de água deu-se após a água atingir 92-95 $\mathrm{C}^{\mathbf{o}}$. Os provadores iniciaram as avaliações quando a temperatura das xícaras atingisse os $55 \mathrm{C}^{\circ}$, respeitando o tempo de 3 a 5 minutos para a degustação após a infusão 
(SCAA, 2013). O estudo sensorial foi conduzido com 06 provadores treinados e certificados como Q-Grader's, conforme proposição de Pereira et al. (2016), Pereira et al., (2018).

\section{Análises físicas e químicas e preparo das amostras 1 Extrato aquoso}

Para realização da análise de extrato aquoso, pesou-se $2 \mathrm{~g}$ da amostra de café torrado em um béquer de $50 \mathrm{~mL}$. Transferiu-se a amostra para um Erlenmeyer de $500 \mathrm{~mL}$ e acrescentou-se $200 \mathrm{~mL}$ de água quente. A solução foi deixada em ebulição por uma hora em chapa de aquecimento. Ainda quente, a solução foi transferida para um balão volumétrico de $500 \mathrm{~mL}$, sendo que o frasco foi lavado com $100 \mathrm{~mL}$ de água quente para transferência de toda a amostra para o balão. O balão foi resfriado e teve seu volume completado com água. A solução foi então filtrada para um frasco Erlenmeyer de $500 \mathrm{~mL}$. Pipetou-se $50 \mathrm{~mL}$ do filtrado em um béquer de $100 \mathrm{~mL}$, previamente tarado em estufa a $105^{\circ} \mathrm{C}$ e pesado. O filtrado foi evaporado em banho-maria até a secagem e posteriormente aquecido em estufa a $105^{\circ} \mathrm{C}$ por uma hora. $\mathrm{O}$ extrato foi resfriado em dessecador e pesado (IAL, 2008).

\section{Cafeína}

Pesou-se $1 \mathrm{~g}$ de amostra de café moído em um béquer de $100 \mathrm{~mL}$. Para evitar a formação de grumos, adicionou-se $4 \mathrm{~mL}$ de ácido sulfúrico. A mistura foi homogeneizada e aquecida em banhomaria durante 15 minutos, agitando a cada 5 minutos. Cuidadosamente, foram adicionados $50 \mathrm{~mL}$ de água quente, deixando a mistura em banho-maria por mais 15 minutos. A mistura foi filtrada a quente para um funil de separação de $500 \mathrm{~mL}$, por meio de papel de filtro quantitativo umedecido com água. Lavou-se o béquer e o filtro com porções de $10 \mathrm{~mL}$ de água quente acidulada três vezes. $\mathrm{O}$ filtrado foi esfriado. Adicionou-se $30 \mathrm{~mL}$ de clorofórmio no filtrado e agitou-se por dois minutos. Esperou-se a separação das camadas e então a camada do clorofórmio (inferior) foi decantada por meio de papel de filtro quantitativo umedecido com clorofórmio. Um balão de fundo chato de $300 \mathrm{~mL}$ recebeu o decantado. Repetiu-se a extração com mais três porções de $30 \mathrm{~mL}$ de clorofórmio. $\mathrm{O}$ extrato de clorofórmio obtido foi evaporado em rotavapor. O resíduo foi dissolvido com água quente e filtrado para um balão volumétrico de $1000 \mathrm{~mL}$. Após esfriar, completou-se o volume do balão com água. Mediu-se a absorbância a 274 nm, em espectrofotômetro e a quantidade de cafeína correspondente foi obtida com auxílio de uma curva-padrão previamente estabelecida (IAL, 2008).

\section{Acidez titulável e pH}

O extrato utilizado para obtenção do $\mathrm{pH}$ e acidez titulável foi preparado a partir de $2 \mathrm{~g}$ de café moído diluído em $50 \mathrm{~mL}$ de água destilada e submetido à agitação por 1 hora em agitador mecânico a 150 rpm. A solução extrato foi filtrada em filtro de papel quantitativo e o pH foi medido por peagâmetro 
digital. Uma alíquota de $5 \mathrm{~mL}$ do filtrado foi diluída em $50 \mathrm{~mL}$ de água destilada. A acidez titulável total foi determinada por titulação com $\mathrm{NaOH} 0,1 \mathrm{~N}$, utilizando 2 - 3 gotas de uma solução de fenolftaleína $1 \%$ como indicador e expressa em $\mathrm{mL}$ de $\mathrm{NaOH} 0,1 \mathrm{~N}$ por $100 \mathrm{~g}$ de amostra (AOAC, 1990).

\section{Análise estatística dos dados}

Foram realizadas análises de componentes principais para agrupar os cafés a partir das características sensoriais e físicas e químicas, mediante exames visuais em dispersões gráficas. Para as análises estatísticas foi utilizado o programa SPSS, versão 22, sendo considerados os valores médios das análises físicas e químicas e sensorial.

\section{RESULTADOS E DISCUSSÃO}

O primeiro gráfico apresenta a dispersão dos cafés oriundos das análises sensoriais realizadas pelos Q-graders. Na Figura 1, pode-se observar que a dispersão dos cafés com base nas coordenadas relativas aos dois primeiros componentes principais $(\mathrm{CP}), \mathrm{CP} 1$ e $\mathrm{CP} 2$, que formaram 3 grupos distintos, sendo que os dois componentes absorveram 92,22\% da variação existentes nas características originais, sendo o CP1 com $75,74 \%$ e o CP2 com $14,83 \%$.

Identificam-se as variáveis nota global e overall (impressão pessoal), com maiores correlações com CP1, 0,9965, 0,9064 e 0,9031, respectivamente, e com maiores coeficientes de ponderação associados ao CP1, são consideradas como as mais importantes para este componente. Por outro lado, as variáveis doçura, ausência de defeitos e uniformidade, com maiores correlações com o CP2, 0,6297, 0,6297 e 0,6280, respectivamente, e com maiores coeficientes de ponderação associados ao CP2, são consideradas como as mais importantes para este componente. 


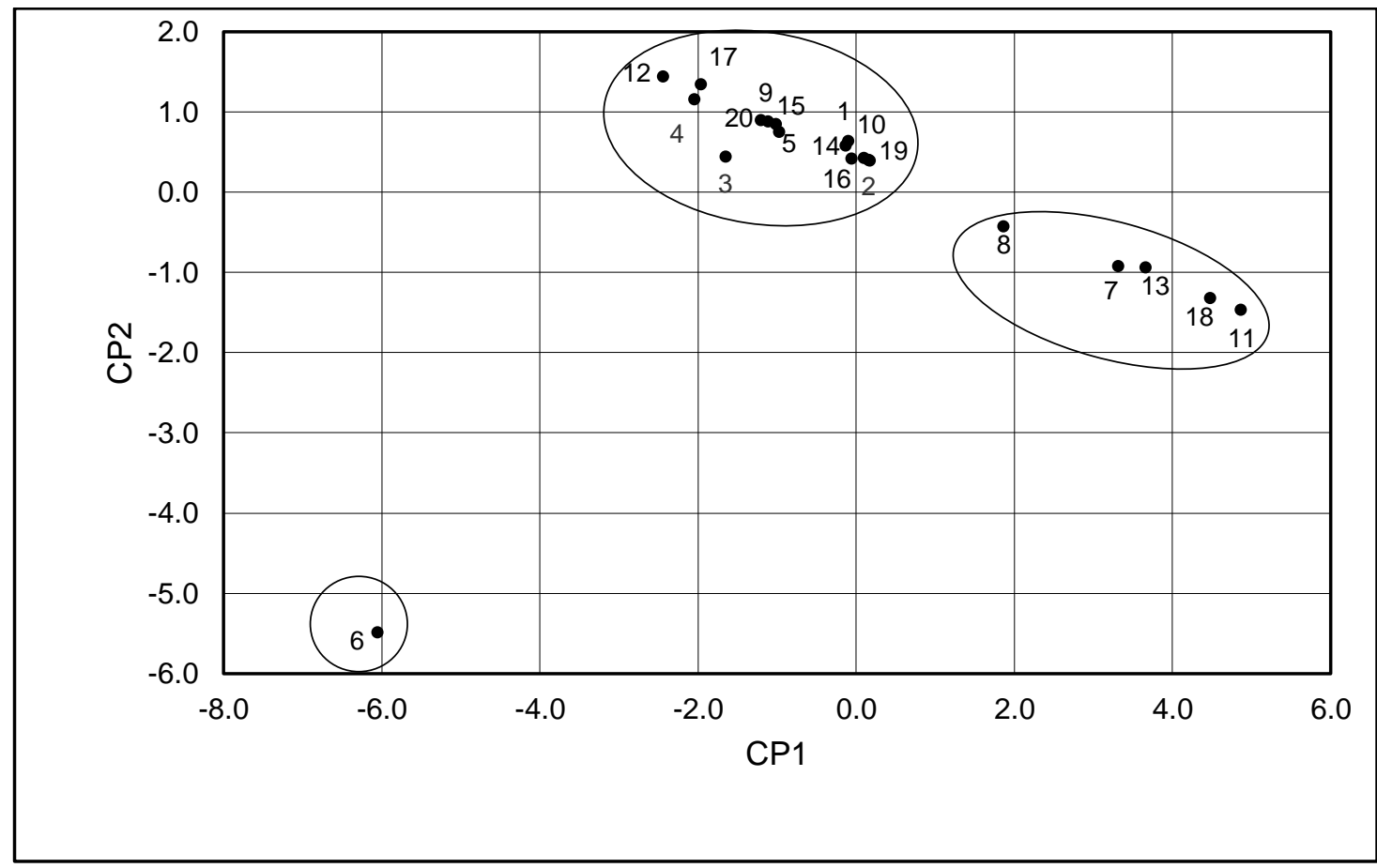

Figura 1 - Diagrama de dispersão em relação aos dois primeiros componentes principais dos 20 cafés, obtido a partir das características sensoriais.

A figura 1 apresenta um dos três grupos formado pelos cafés $8,7,13,18$, 11, indicando um agrupamento em relação aos os cinco primeiros colocados do concurso de qualidade, ou seja, na percepção sensorial, o resultado da análise multivariada indica um agrupamento dos melhores cafés, com base na similaridade do PCA.

Os resultados na figura 1 indicaram três agrupamentos, sendo os cafés excepcionais e com notas acima dos 86 pontos, seguido das amostras $(8,7,13,18$ e 11), seguido do segundo grupo com a amostra 6 (café com defeito), e finalizada pelo terceiro grupo, amostras de (12, 4, 17, 20, 9, 3, 15, 5, $14,1,16,10,19$ e 2 ) com cafés apenas especiais que variaram de 82 a 84 pontos na nota global.

Outro grupo formado pelo café 6 indicou resultados sensoriais com presença de xícara com defeito (riada), indicando baixa qualidade do café. Esse resultado abre duas formas de entendimento, a primeira é que a observação de uma xícara com defeito no café pode ter sido oriunda da transformação química que ocorre durante o preparo, torra, moagem e análise sensorial, fazendo com que os Q-Graders julgassem o paladar como repugnante, ou que a contaminação tenha ocorrido por conta de um fruto defeituoso, modificando o padrão de qualidade. Tais interações entre a descrição de um composto por Q-Graders são complexas de serem completamente descritas. 
Isso sugere que os receptores especializados pelos neurônios, agrupados em cavidades no interior do paladar, geram o gosto e as percepções (gustativas), nomeadamente doce, azedo, salgado e amargo. Terminações nervosas livres espalhadas por toda a cavidade bucal geram a sensação na boca e provocam tais percepções, as combinações destas sensações com as do nariz produzem a percepção de sabor (SÁENZ-NAVAJAS et al., 2010). A percepção do gosto por uma pessoa resulta de uma combinação dos estímulos das células do palato que compõem o paladar. Estes compostos correspondem a quatro sensações de "azedo" (concentração de íons de hidrogênio), "sal" (íons de sal), "doce" (compostos orgânicos) e "amargo" (vários tipos de compostos, incluindo alcalóides e glicosídeos). A diferenciação por sabor de alimentos individuais baseia-se, até certo ponto, no equilíbrio entre estes quatro aspectos, sendo o aroma o mais impactante para a tomada de decisão (MCCORKINDALE \& MCCORKINDALE, 2000).

Desta forma, os resultados indicam que os Q-Graders foram capazes de formular o agrupamento de 03 grupos distintos de cafés, os cafés acima dos 86 pontos tidos como excepcionais pelo protocolo da SCAA, cafés considerados especiais e um café como não especial. Estes resultados assemelhamse aos resultados de Chollet \& Valentin (2001). Nesse estudo, os provadores de cerveja treinados e não treinados tiveram a mesma precisão, variando apenas na terminologia, ou seja, é comum que provadores de alimentos façam agrupamentos sensoriais com base em características de similaridades sensoriais, como observado nos resultados deste estudo.

Kleinwächter e Selmar (2010); Joët et al. (2010), discutem que a formação de aromas e sabores são afetados diretamente pelo manejo, clima e altitude das lavouras, podendo ocasionar no surgimento de compostos que formam sabores agradáveis ou desagradáveis ao paladar e que muitos destes compostos são difíceis de serem distinguidos. Esses fatores que tornam a análise sensorial tão complexa e distinta das demais técnicas analíticas, pois além de lidar com as interações do meio ambiente onde o produto é produzido, os Q-Graders precisam interpretar e distinguir o produto em classes que sejam aceitas como produtos especiais ou não especiais, para que a indústria os coloque a disposição do consumidor.

Sobre essa amostra, é necessário enfatizar que a bebida classificada como "riada" possui como característica leve sabor de iodofórmio ou ácido fênico, (PINTO, 2001). Porém os cafés submetidos ao estudo possuíam qualidade superior aos 80 pontos conforme protocolo da SCAA, esse fator indica e reforça as variações químicas que podem ocorrer entre as análises e entre avaliadores, ou seja, o café que apresentou uma xícara com defeito (riada) foi preterida pelos Q-Graders indicando 
que existem outros parâmetros que devem ser monitorados entre o preparo das amostras, a torra e a análise sensorial.

Os resultados das análises físicas e químicas, podem ser expressos na figura 2, pode-se observar que a dispersão dos cafés com base nas coordenadas relativas aos dois primeiros componentes principais, $\mathrm{CP} 1$ e $\mathrm{CP} 2$, que formaram 8 grupos distintos e que os dois componentes absorveram $72,27 \%$ da variação existentes nas características originais, sendo o CP1 com 43,40\% e o CP2 com 28,87\%. Identificam-se as variáveis acidez titulável e pH com maiores correlações com CP1, 0,8908 e 0,8563, respectivamente, e com maiores coeficientes de ponderação associados ao CP1, são consideradas como as mais importantes para este componente. Por outro lado, as variáveis cafeína e extrato solúvel com maiores correlações com o CP2, 0,8201 e 0,6164, respectivamente, e com maiores coeficientes de ponderação associados ao $\mathrm{CP} 2$, são consideradas como as mais importantes para este componente.

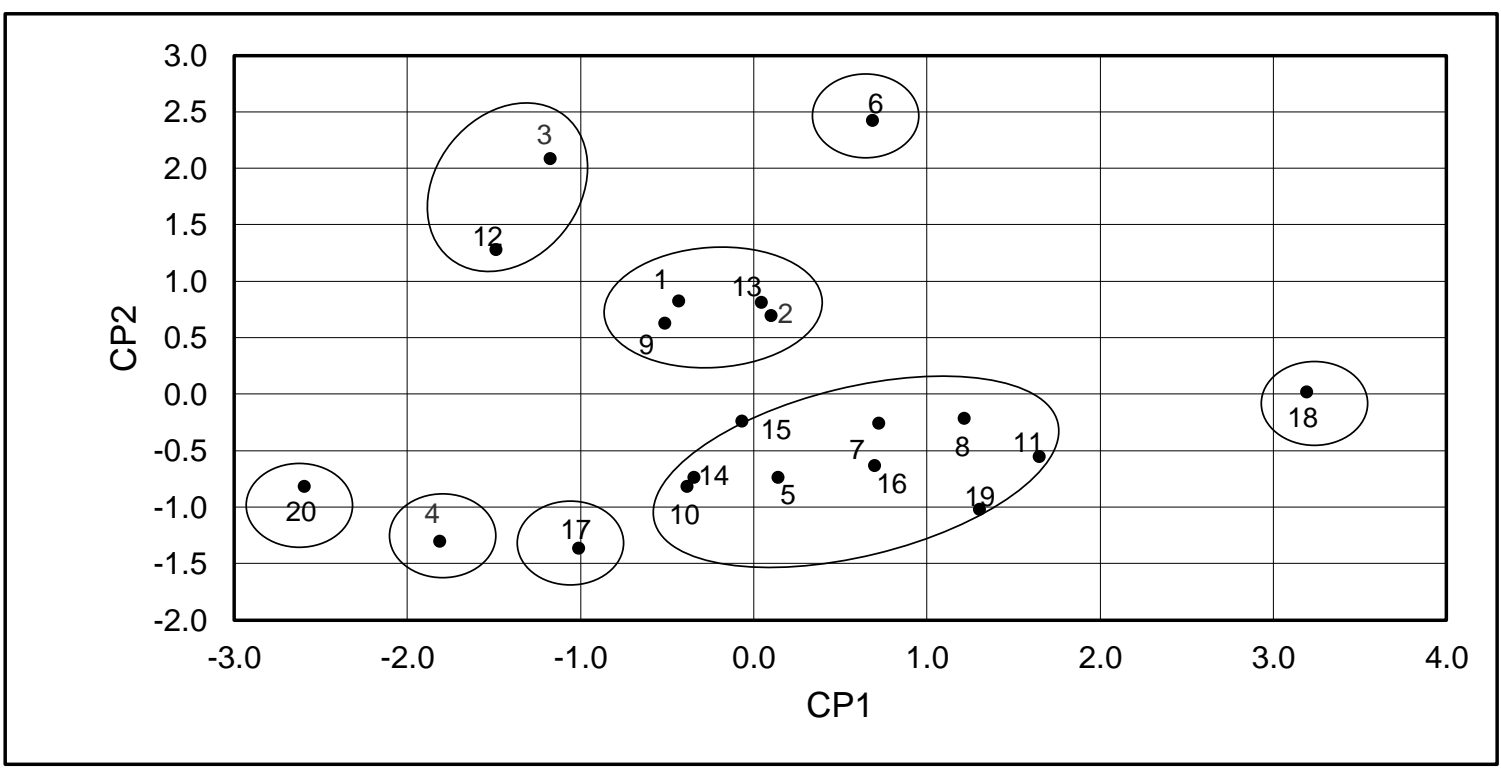

Figura 2 - Diagrama de dispersão em relação aos dois primeiros componentes principais dos 20 cafés, obtido a partir das características físicas e químicas.

Os resultados apresentados na figura 2 indicam que o grupo formado pelos cinco cafés especiais acima dos 86 pontos pela SCAA $(8,7,13,18$ e 11) apresenta-se completamente distintos, ou seja, esse grupo foi desmembrado em relação a análises sensorial.

Quando observado os resultados das análises físicas e químicas, o grupo dos cafés excepcionais na figura 1, composto pelas amostras $(7,8,11,13$ e 18), desmembram-se em três outros grupos, ficando o café 18 isolado, seguido do grupo $(1,13,9$ e 2), do grupo $(8,7,11,10,14,15,5,7$, 16 e 19). A amostra com defeito sensorial na figura 2 segue o mesmo comportamento na figura 1 , ou 
seja, agrupa-se distintamente. Sendo os demais grupos isolados compostos pelas amostras, 20, 4 e 17.

Esses constituintes químicos são responsáveis pela formação de compostos ligados ao sabor e aroma que o café apresenta durante a sua degustação (RIBEIRO et al, 2013). Siqueira et al. (2005) descrevem que a acidez percebida no café é um atributo importante para a análise sensorial do produto, sabendo que sua intensidade varia conforme o estádio de maturação dos frutos, local de origem, tipo de colheita, forma de processamento, tipo de secagem e condições climáticas durante a colheita e secagem. Complementar à acidez do café, o pH é um indicativo de eventuais transformações dos frutos de café. Desta forma, os dados observados na figura 2 estão de acordo com as proposições de Siqueira et al., (2005).

Com base nos resultados das figuras 1 e 2, observa-se que as análises de agrupamento entre as variáveis físicas e químicas não possuem as mesmas similaridades entre os cafés em relação as variáveis sensoriais entre os PCA.

As recentes discussões sobre a importância de mesclar técnicas para aferir maior fidedignidade ao processo de análise da qualidade do café, tendo em vista as possíveis falhas que podem ocorrem no processo de análise sensorial, foram sugeridas por Munõz (2002), Seo et al. (2008); Alvarado \& Linnemann (2010); Di Donfrancesco et al. (2014). Porém, os resultados desta pesquisa, indicam que a complexidade de interpretar e entender a estrutura qualitativa de um alimento, a partir de análises físicas e químicas é tão densa quanto à subjetividade por trás da análise sensorial. Isso sugere certa dificuldade em se correlacionar dados sensoriais com análises físicas e químicas. Sugere-se então que sejam inseridas técnicas mais refinadas como cromatografia líquida ou gasosa, para que se possa entender a fundo quais ácidos orgânicos, compostos voláteis ou até mesmo outras estruturas químicas se correlacionam com as interpretações sensoriais descritas pelos Q-graders.

Seria mais plausível a combinação de avaliação sensorial com a análise instrumental (infravermelho e ressonância magnética nuclear, etc.) para fornecer mais informações sobre pontos extremos, como observado nas amostras 6 e 18, gerando uma imagem mais completa do produto (ROSS, 2009). Para pesquisas futuras, sugere-se que as características químicas do café devem ser entendidas através de parâmetros mais robustos, oriundos de análises mais refinadas, tanto a nível químico, quanto sensorial. 


\section{CONCLUSÕES}

Os resultados não apresentaram consistência entre similaridade de agrupamento dos cafés, baseados nas características sensoriais em relação aos resultados das características físico-químicos.

Análises sensoriais, físicas e químicas colaboraram para o agrupamento de casos extremos, cafés excepcionais e café defeituoso, ainda assim a análise físicas e químicas demonstram-se limitadas para descrever a qualidade real. Sugerindo assim foco em técnicas mais refinadas que possam indicar padrões mais confiáveis na definição da qualidade do café.

As variáveis sensoriais de nota global e overall (impressão pessoal) seguida das variáveis acidez titulável e pH, oriundas das análises físicas e químicas são responsáveis de forma mais significativa para formação dos agrupamentos entre dados. Isso sugere que os ácidos orgânicos devem ser melhor compreendidos para que seja possível correlacionar de forma mais precisa os fatores químicos com respostas sensoriais de Q-Graders.

Apesar de subjetiva, a análise sensorial com emprego do protocolo da SCAA constitui-se em uma técnica eficiente para determinação da qualidade final do café.

\section{AGRADECIMENTOS}

Os autores agradecem ao Instituto Federal do Espírito Santo pelo suporte neste projeto, aos QGraders participantes que se dedicaram à realização do estudo. Agradecem também ao CNPq pela viabilização de recursos via projeto ( $\left.n^{\circ} 469058 / 2014-5\right)$ para condução desta pesquisa.

\section{REFERÊNCIAS}

ALVARADO, R. A.; LINNEMANN, A. R. The predictive value of a small consumer panel for coffee-cupper. British Food Journal, Judgment, v.112, n.9, p.1023-1032, 2010.

ASSOCIATION OF OFFICIAL ANALYTICAL CHEMISTS. Official Methods of Analysis of the Association of Official Analytical Chemists. v. 2. 15.ed. Washington, 1990.

BHUMIRATANA, N.; ADHIKARI, K.; CHAMBERS, E. Evolution of sensory aroma attributes from coffee beans to brewed coffee. LWT - Food Science and Technology, v. 44, n.10, p.21852192. 2011.

CLEMENTE, A.C.S.; CIRILLO, M. A.; MALTA, M. R.; CAIXETA, F.; PEREIRA, C.C.; DA ROSA, S.D.V.F. Operações pós-colheita e qualidade físico-química e sensorial de cafés. Coffee Science, Lavras, v.10, n.2, p.233-241, 2015. 
CHOLLET, S.; VALENTIN, D. Impact of training on beer flavor perception and description: are trained and untrained subjects really different? Journal of Sensory Studies, v.16, n.6, p.601-618, 2001.

DI DONFRANCESCO, B.; GUZMAN, N.G.; CHAMBERS, E. Comparison of results from cupping and descriptive sensory analysis of Colombian brewed coffee. Journal of Sensory Studies, v.29, n.4, p.301-311, 2014.

FEIRA-MORALES, A.M. Examining the case of green coffee to illustrate the limitations of grading systems/expert tasters in sensory evaluation for quality control. Food Quality and Preference, Londres, v.13, n.6, p.355-367, 2002.

INSTITUTO ADOLFO LUTZ - IAL. Métodos físico-químicos para análise de alimentos. 4. ed. São Paulo: Instituto Adolfo Lutz, 2008.

JOËT, T.; LAFFARGUE, A.; DESCROIX, F.; DOULBEAU, S.; BERTRAND, B.; KOCHKO, A.; DUSSERT, S. Influence of environmental factors, wet processing and their interactions on the biochemical composition of green Arabica coffee beans. Food Chemistry, v.118, n.3, p. 693-701, 2010.

KLEINWÄCHTER, M.; SELMAR, D. Influence of drying on the content of sugars in wet processed green Arabica coffees. Food Chemistry, v.119, n.2, p.500-504, 2010.

MCCORKINDALE, L.M.; MCCORKINDALE, L.M. What Is Taste? Nutrition \& Food Science, v.6, n.1, p.8-12, 2000.

MOREIRA, R. F. A.; TRUGO, L.C. Componentes voláteis do café torrado. Parte II. Compostos alifáticos, alicíclicos e aromáticos. Química Nova, São Paulo, v.23, n.2, p.2, 1999.

MUNHÕZ, A. M. Sensory evaluation in quality control: an overview, new developments and future opportunities. Food Quality and Preference, v.13, n.6, p.329-339, 2002.

PEREIRA, M.C.; CHALFOUN, S.M.; CARVALHO, G. R.; SAVIAN, T.V. Multivariate analysis of sensory characteristics of coffee grains (Coffea arabica L.) in the region of upper Paranaíba. Acta Scientiarum. Agronomy, Maringá, v. 32, n. 4, p.635-641, 2010.

PEREIRA, L. L. et al. Tamanho ótimo do número de Q-Graders de café com uso do protocolo SCAA. In.: SIMPOSIO INTERNACIONAL DE INGENIERÍA INDUSTRIAL: ACTUALIDAD Y NUEVAS TENDENCIAS, 9., 2016. Porto Alegre, Brasil. Anais... Porto Alegre, 2016.

PEREIRA, L.L. Novas abordagens para produção de cafés especiais a partir do processamento via-úmida. 2017. 200f. Tese de Doutorado (Departamento de Engenharia de Produção) Universidade Federal do Rio Grande do Sul. Porto Alegre, Rio Grande do Sul, 2017.

PEREIRA, L.L.; GUARÇONI, R.C.; CARDOSO, W.S.; TAQUES, R.C.; MOREIRA, T.R.; SILVA, S.F.; CATEN, C.S.T. Influence of Solar Radiation and Wet Processing on the Final Quality of Arabica Coffee. Journal of Food Quality, Article ID 6408571, 9p. 2018. (Disponível em: <http: https://www.hindawi.com/journals/jfq/2018/6408571/>. Acesso em: mar.2018. 
PINTO, N.A.V.D.; FERNANDES, S.M.; PIRES, T.C.; PEREIRA, R.G.F.A.; CARVALHO, V.D. Avaliação dos polifenóis e açúcares em padrões de bebida do café torrado tipo expresso. Revista Brasileira de Agrociência, Pelotas, v.7, n 3, p.193-195, 2001.

SEO, H. S., LEE, S. Y., HWANG, Y. Development of sensory attribute pool of brewed Coffee. Journal of Sensory Studies, v.24, n.1, p.111-132, 2010.

RIBEIRO, B.B.; MENDONÇA, L.M.V.L.; ASSIS, G.A.; MENDONÇA, J.M.A.; MALTA, M.R. Avaliação química e sensorial de blends de Coffea canephora pierre e Coffea arabica L. Coffee Science, Lavras, v.9, n.2, p.178-186, 2014.

ROSS, C.F. Sensory science at the human e machine interface. Trends in Food Science \& Technology, Amsterdam, v.20, n.2, p.63-72, 2009.

SÁENZ-NAVAJAS, M.P.; FERREIRA, V.; DIZY, M. \& FERNÁNDEZ-ZURBANO, P. Characterization of taste-active fractions in red wine combining HPLC fractionation, sensory analysis and ultra-performance liquid chromatography coupled with mass spectrometry detection. Analytica Chimica Acta, v.673, n.2, p.151-159, 2010.

SCHOLZ, M.B.S.; SILVA, J.V.N.; FIGUEIREDO, V.R.G.; KITZBERGER, C.S.G. Atributos sensoriais e características físico-químicas de bebida de cultivares de café do Iapar. Coffee Science, Lavras, v.8, n.1, p.6-16, 2013.

SIQUEIRA, H. H.; ABREU, C. M. P. Composição físico-química e qualidade do café submetido a dois tipos de torração e com diferentes formas de processamento. Ciência Agrotecnologia, Lavras, v.30, n.1, p.112-117, 2005.

SCAA. Specialty Coffee Association of American. Protocols. January, 23, 2013. Disponível em: < http://www.scaa.org/PDF/resources/cupping-protocols.pdf $>$.

TOCI, A.T.; FARAH, A. Volatile fingerprint of Brazilian defective coffee seeds: corroboration of potential marker compounds and identification of new low-quality indicators. Food Chemistry, v.153, p.298-314, 2014.

TOCI, A.T.; FARAH, A. Volatile compounds as potential defective coffee beans' markers. Food Chemistry, v.108, n.3, p.1133-1141, 2008. 